\title{
Application of Bruner's Learning Theory in Mathematics Studies
}

\author{
Ping Wen \\ School of Mathematics and Information Technology \\ Yuxi Normal University \\ Yuxi, China 653100
}

\begin{abstract}
Bruner is a famous educational psychologist and cognitive psychologist in the United States. This paper mainly introduces Bruner's cognitive structure learning theory and discovery learning theory. Through the analysis of mathematics teaching cases, this paper discusses how to apply Bruner's learning theory in mathematics teaching. The purpose of mathematics teaching is to enable students to master the knowledge structure of mathematics in a comprehensive way. Mathematics teachers should actively create conditions in teaching, and guide students to discover and learn through hands-on activities, thinking and representation. From passive acquisition of knowledge to active discovery and independent inquiry, students become the main body of mathematics studies.
\end{abstract}

Keywords-Bruner; learning theory; mathematics studies

\section{INTRODUCTION}

Jerome Seymour Bruner, born in New York in 1915, is an American educational psychologist and cognitive psychologist. Bruner has done a lot of research on cognitive processes, has written many books on word learning, concept formation and thinking, and has made outstanding contributions to the systematization and scientization of cognitive psychological theory. He is a pioneer of cognitive psychology and a typical representative of the practice of psychological principles in education, who is praised as the person who has the greatest influence on American education after Dewey. Bruner assimilated the German Gestalt psychology and the Swiss Piaget (J.) developmental psychology. On the basis of criticizing and inheriting Dewey $\mathbf{J}$ educational thought and with his long-term research, he gradually formed the model and theory of "discovery learning". [1]

His main representative works on education are: Educational Process (1960), The Relevance of Education (1971), and Teaching Theory Discussion (1966). And the Educational Process is a book which expresses the view of knowledge according to structuralism and the process of cognition according to perception, while the latter two are complementary explanations of certain ideas in the educational process. These three books represent Bruner's main educational viewpoints, of which Educational Process is the most important and influential one. Bruner also has some research on mathematics teaching, and most of the ideas in his educational works are supported by mathematical cases. Lefthanded Thinking-Intuition Ability, Emotion and Spontaneity
(1962) is a book whose most articles are the ones on lefthanded thinking. The section "On Mathematical Learning" is an article about right-handed thinking. This section is a transcript of his conversation with his stepdaughter Lynn about how to teach students to think mathematically. [2]

\section{BRUNER'S LEARNING THEORY}

\section{A. Cognitive Structure Learning Theory}

Bruner believes that the essence of learning is that one connects the similar things and organizes them into meaningful structures, and learning is the organization and reorganization of cognitive structures. Knowledge learning is to form the knowledge structure of all subjects in the minds of students. Bruner holds that cognitive structure is a general way for people to perceive and generalize the external physical world, and it is a psychological structure formed in the process of human activities to recognize the external things. Cognitive structure is progressive and multi-level, developing from low level to advanced level. And it is formed on the basis of past experience and is constantly changing in the process of learning. In addition, the formation of cognitive structure is an important internal factor and foundation for further learning and understanding of new knowledge.

Bruner calls cognitive structure "representation" and holds that representation can be divided into three types: action representation, image representation and symbolic representation. The so-called behavioral characteristics mainly refer to relying on action to perceive the world, for example, a two-year-old infant often relies on action to perceive the world. As children grow older, they begin to use visual and auditory representations or images in their minds to represent external things and try to solve problems through images. We call this representation as image representation. From the age of about six or seven, individuals can use symbols such as language and numbers to represent experience, while using these symbols to learn and gain experience. We call this representation as symbolic representation. The three representations do not exist in isolation. As the individual develops to a certain stage, the three representations coexist in individual cognitive structure, complement each other and work together on cognitive activities.

Bruner thought that knowledge learning is to form certain knowledge structure in the minds of students. This knowledge 
structure is made up of the basic concepts, basic ideas or principles of subject knowledge. The structural form of knowledge structure is made up of human coding system.

Bruner outlines the general process of cognition. He believes that learning a subject or a point of knowledge involves three almost simultaneous processes: acquisition of new knowledge, transformation and evaluation of knowledge. The so-called acquisition of new knowledge refers to the process in which new knowledge is connected with existing knowledge, experience and cognitive structure. It is a process of active understanding, which brings new knowledge into the existing knowledge structure through "assimilation" or "adaptation". The acquisition of new knowledge is the most important link in the cognitive process. In order to acquire new knowledge when learning, students need to establish the relationship between the old and the new knowledge so as to form a new cognitive structure. The transformation of knowledge is the further analysis and generalization of new knowledge, which can be transformed into another form to adapt to new tasks. Comment: It is a test of knowledge transformation to see whether the analysis and generalization of knowledge is appropriate and the operation is correct.

In the process of learning geometry knowledge, students manifest Bruner's cognitive structure learning theory very well. Taking the learning of square knowledge as an example, this paper expounds how cognitive structure theory can help students master the knowledge. In order to build knowledge structure chart, we first need to analyze the concept of square. Then the concepts of quadrangle, parallelogram, rectangle and rhombus related to square are defined. A quadrilateral is defined as a closed plane figure or a three-dimensional figure formed by four uncrossed sides which are not on the same line and are connected by the end of each line in turn. A square is defined as a quadrilateral whose four sides are equal and four angles are right angles. From this definition, we can see that a square belongs to a special quadrilateral. We call a quadrilateral "generic concept" and a square "species concept". The difference between a square and a quadrilateral is that when the four sides of a quadrilateral are equal and one of the angles is a right angle, a square is formed. We call the difference between the "generic concept" and "species concept" as "species difference". The definition of a square is a method using "genus" and "species difference" in mathematical concepts to define new concepts. In the same way, we can analyze the concepts of parallelogram, rectangle and diamond. It can be found that a series of geometric concepts are defined in this way. So there is a close relationship between the above mathematical concepts. A quadrangle is the most one containing the widest range. If the quadrangle satisfies that two sets of opposite sides are parallel, then a parallelogram is formed. A rectangle is formed if a parallelogram has a right angle, and a square is formed if four sides are equal. Similarly, if a parallelogram has a set of equal adjacent sides, a diamond is formed; and a square the diamond has a right angle. By analyzing the relationship between various mathematical concepts, a quadrilateral knowledge structure diagram can be drawn, as shown in "Fig. 1 ".

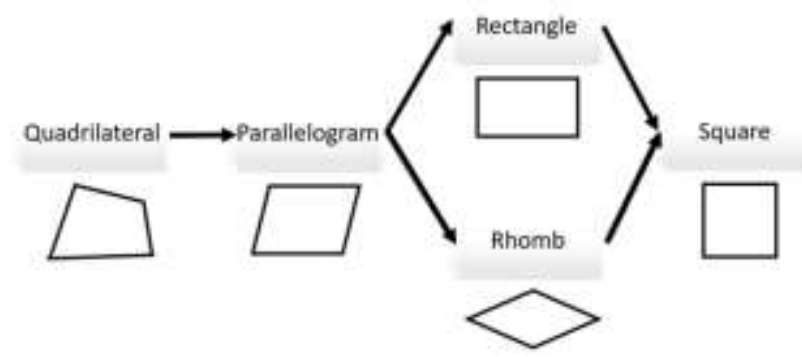

Fig. 1. Knowledge structure diagram of quadrilateral.

When students learn the knowledge of square, they do not grasp the concept and nature of square in isolation, instead link the knowledge of orthomorphism to other geometric knowledge, such as quadrilateral, parallelogram, rectangle, diamond, and so on. And they conclude the new knowledge into the original knowledge structure, so as to form and perfect the quadrilateral knowledge structure diagram constantly in the mind. The role of teachers is to help students establish connections between new and old knowledge, let students know something about the quadrilateral content, and clear the internal relationship between various geometric concepts, so as to establish a complete knowledge system of knowledge points. "No matter what subject we choose to teach, we must make sure that students understand or master the basic structure of the subject," [3] Bruner said. Mathematics learning is to link the similar things and organize them into meaningful structures. Learning is the arrangement and rearrangement of cognitive structure. In the process of teaching, teachers should consciously help students to establish the connection between knowledge points, and form the knowledge structure of mathematics in students' minds.

\section{B. Discovery of Learning Theory}

In terms of teaching method, Bruner put forward the "discovery learning method". "Discovery is not limited to the search for unknown things, but rather it includes all the means of obtaining knowledge through one's own mind," Bruner said. Bruner's "discovery" is not a scientist's invention, but "an activity in which students organize what they know in their own way rather than books". [4] What he calls discovery learning is a process in which students acquire new knowledge for them by reading books and literature independently and thinking independently. Bruner attaches great importance to discovery and believes that students are not passive or passive recipients, but active explorers. Cognitive process is a process in which people mainly choose, transform, store and apply the things that come into their senses, in which people actively study, adapt to and transform the environment. He suggested that teachers should provide more materials for students to analyze and synthesize the deserved conclusion rules and become "discoverers". In this way, we can better explore the potential of wisdom, arouse the enthusiasm of students' thinking, stimulate students' excitement, self-confidence and interest in learning, and help to maintain memory.

Bruner and his collaborator, mathematician Z.P. Dienes, designed such a classroom experiment in mathematics to teach eight-year-olds the factorization of quadratic equation. 
Factorization is a junior high school teaching content, which is difficult for 8-year-old children. Bruner uses discovery learning method to guide students to learn. From this case, we can see how the discovery learning mode is applied in classroom teaching practice.

Firstly, the teacher introduces three different kinds of wood chips to the students. The first one is a large square with the side length of $x$, which is called as " $x^{2}$ "square; the second one is a rectangle with side lengths of 1 and $x$, called as " $1 \square x$ " or " $x$ " rectangle; the third is a small square with the side length of 1 , called as "1.1" or "1" square, as shown in "Fig. 2". In the classroom, teachers provide students with more than three pieces of wood. After the introduction of rules, the students can piece them together freely so as to obtain the corresponding perceptual experience.

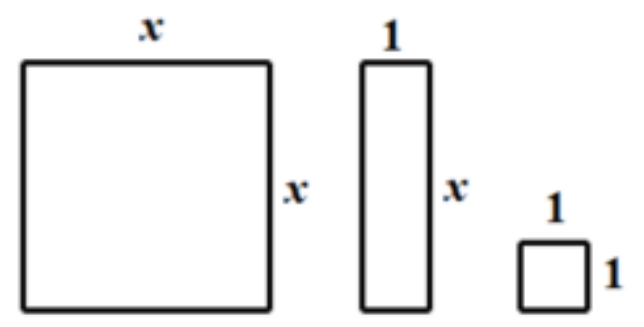

Fig. 2. Three different kinds of wood chips.

The teacher began to ask, "Can you use these pieces of wood to make a square larger than the" $x^{2}$ "? With a little effort, the students finished the task easily by fiddling with wood chips. They made a bigger square with three different wooden chips, as shown in "Fig.3".
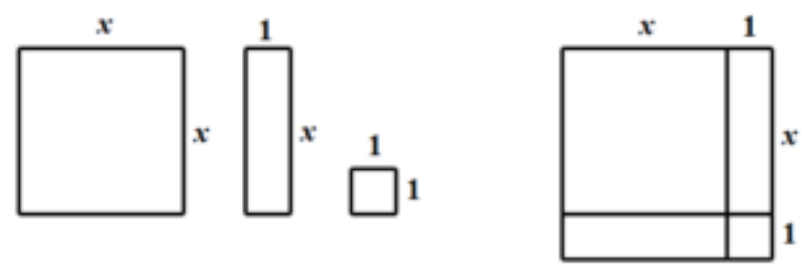

Fig. 3. Wood puzzle results by using three pieces

Next, the teacher asked students to describe how they had done the work of making bigger squares with three pieces of wood. Students observe, think and discuss, while teachers give hints or questions according to the actual situation. Most students concluded that "the large square that we put together is a $x^{2}$ square plate with two ' $x$ ' plates and a ' 1 ' plate." The teacher then guides the students to record the representation in marking, that is, the " $x^{2}$ square" is represented by " $x^{2}$ " and plus is "+". Finally the expression of " $x^{2}+2 x+1$, can be formed. With discussing and thinking, students will have another way to describe their work. This method is considered from the side of square, that is, $(x+1)(x+1)$. Since the two representations represent the same square, they can be equated and an equation $x^{2}+2 x+1=(x+1)(x+1)$ is formed. So far, the factorization mode of quadratic equation of one variable has been "discovered" in the process of students' hands-on operation.

After acquiring such knowledge and methods, teachers guide students to constantly apply them to new problem situations, that is, constantly making larger squares and establishing new equations in accordance with the above ways and means. As shown in "Fig. 4", with three pieces of wood to make bigger squares, students may get two different situations. But in any case, the formula of mathematics can be summed up to $x^{2}+4 x+4=(x+2)(x+2)$.
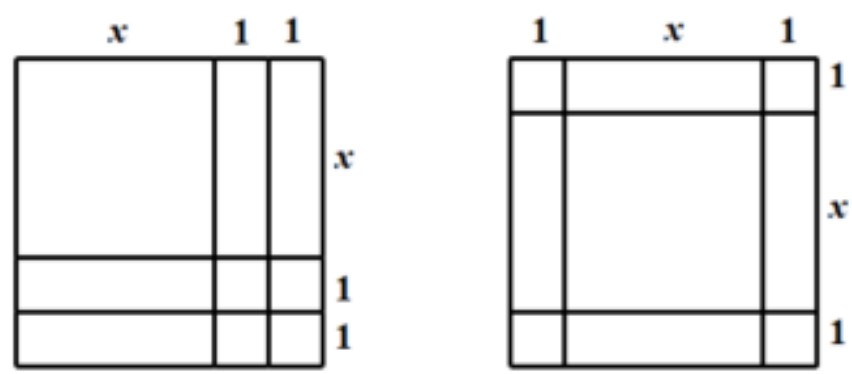

Fig. 4. Two puzzle situations with three pieces of wood.

And then teachers guide the students to operate, using three pieces of wood to make a bigger square, as shown in "Fig.5". Students operate first, thinking about how to operate, and finally summarize the operation as mathematical expressions. Although there are many ways of puzzle, they can be expressed in $x^{2}+6 x+9=(x+3)(x+3)$. After several hands-on operations and marking, comparing with the mathematical formulas summarized, students finally discovered the factorization rule of quadratic equation with one unknown in algebra.

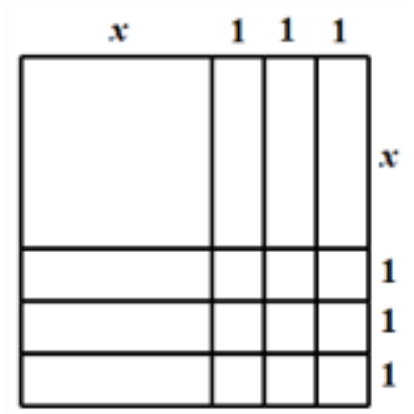

Fig. 5. Using three wood chips to make a bigger square.

Bruner's discovery learning method is embodied very well in the above cases. The teacher's role is to create a situation in which students can explore independently, rather than to provide ready-made knowledge. Instead of memorizing what is said in teachers and textbooks, the main purpose of learning is students' participation in the process of establishing the knowledge system of the discipline. Bruner's discovery learning emphasizes the process of inquiry, emphasizing the cultivation of students' spirit of exploring problems and the ability to solve problems independently. Mathematics learning requires students to rely on their own ability to discover the generalization behind a particular mathematical operation. In 
short, it is discovering some laws of mathematics. Bruner believes that the constant alternation of operation and representation is a necessary condition for discovery. [5]

Through Bruner's discovery learning method, we can deduce the area formula of the circle as follows and guide students to find the formula of circle area in hands-on operation. First, teachers provide students with circular pieces of paper with different sizes and ask questions: how to calculate the area of a circle. Students explore it by hands-on operation, and teachers can remind students in this process that the circle can be turned into the geometry you've learned so as to find the area of the circle. Students have many ways to teach, and teachers should guide them. Some students have this method that a circle can be divided into four parts through the circle center O, as the puzzle shown in "Fig.6". The puzzle is close to the parallelogram that students have learned. However, there are still errors, and teachers should guide students how to narrow the errors.
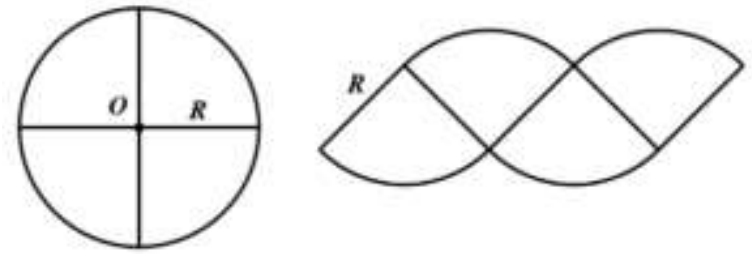

Fig. 6. Dividing the circle into four equal parts for puzzle.

Under the guidance of teachers, it is not difficult for the students to find that if a circle is divided into eight parts, according to the above method of puzzle, the puzzle is closer to the parallelogram. And its error is smaller than that of quartering, as shown in "Fig.7".
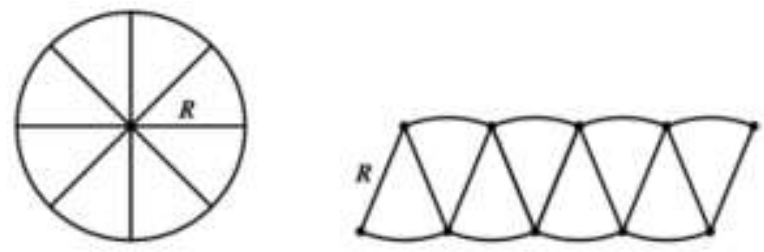

Fig. 7. Dividing the circle into eight equal parts for puzzle.

Based on the above two steps, students have been able to do puzzles according to the above ideas. And students can find that as the number of equal points increases, the error gradually decreases. The puzzles are closer to a rectangle, and the two sides of the rectangle are $R$ and $\pi \cdot R$, as shown in "Fig. 8 ". The formula of a circle area can be deduced: $S=\pi R^{2}$.

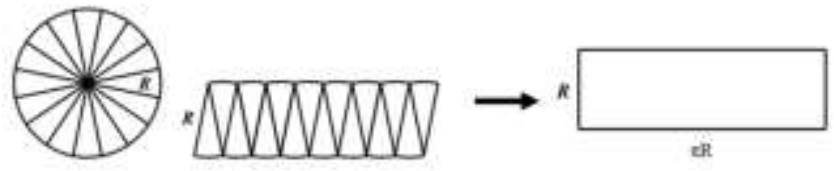

Fig. 8. Transforming the circle approximately into a rectangle.

Through the above two cases it can be seen that in Bruner's discovery learning method, teaching should first allow students to do hands-on operation, and think in operation before the representation. Students should participate in the process of learning and thinking, through a series of discovery behavior to get the content of learning and make a summary. In the whole process, students always actively participate in learning, not passive but active in acceptance of knowledge and "discovery" of what they should learn in the mathematical knowledge.

\section{CONCLUSION}

Bruner's learning theory still has theoretical guiding significance and practical value for today's mathematics teaching. Mathematics teaching should take students' original development characteristics and state as the starting point, give students appropriate tasks, and fully tap the potentials of students, so that every student can reach the maximum development at the original cognitive level. In classroom teaching, the relationship between teachers and students has changed from "authority-obedience" to "value guidance-active participation". Through this process of independent inquiry and joint discovery of knowledge, students acquire a successful experience and realize their sense of self-value. Therefore, teachers should actively create conditions to guide students to change their learning styles. From passive acceptance of knowledge to active discovery and independent inquiry, learning will really become their own independent activities.

\section{REFERENCES}

[1] Zhao Desu. Revelation of Bruner's "Discovery Learning" for quality education inspiration $[\mathrm{J}]$. Journal of Guizhou Educational College (social science), 2003 (4) : 1-4.

[2] Chen Lihua, Dai Qin. Analysis of Bruner's Thoughts on Education [J]. Journal of Inner Mongolia Normal University (education science), 2013 (10) : 73-75. s

[3] (U.S.) Jerome - Bruner, Educational Process [M ] . Translated by Shanghai Normal University Foreign Education Laboratory. Shanghai: Shanghai People' s Publishing House, 1973.

[4] (U.S.) Jerome - Bruner, Selection of Bruner's Education Theory [M] . Translated by Shao Ruizhen, etc. Beijing: People's Education Press, 1989. [5] (U.S.) Jerome - Bruner. Discussion left-Handedness Thought -
Intuitive Abilities, Emotions and Spontaneity [M]. Translated by Peng Zhengmei, Shanghai: Shanghai People' s Publishing House, 2004. 